\title{
Perspectiva sobre modelos y teorías de enfermería en el ámbito de nefrología
}

\author{
Susana Varez Pelaez - María López Parra - Susana Santos Ruiz - Dolors Abril Sabater
}

Corporación Sanitaria Parc Taulí. Sabadell

\section{RESUMEN}

Enfermería depende de la existencia de un conocimiento teórico para todas las áreas. Usar un modelo Conceptual o Teoría de Enfermería en la atención, garantiza que los profesionales compartan igual concepción del metaparadigma y lenguaje en la orientación de los cuidados. Los objetivos del estudio son: 1.Conocer el posicionamiento de los profesionales de enfermería de la unidad nefrológica sobre modelos conceptuales y teorías de enfermería. 2. Conocer el nivel de aplicabilidad sobre los mismos.

Estudio descriptivo observacional a todos los profesionales de enfermería de nuestra unidad. Se recogieron los datos mediante un cuestionario de elaboración propia, realizando un estudio descriptivo de todas las variables, expresando los resultados de las variables cuantitativas en forma de media y desviación estándar. Las variables categóricas se expresaron en forma de frecuencia y porcentajes.

Se analizaron 27 cuestionarios. Índice de respuesta del $77,1 \%$. Los datos sociodemográficos son:

Correspondencia:
Susana Varez
Unidad de Nefrología de la Corporación Sanitaria
Parc Taulí - Parc Taulí s/n
08208 Sabadell (Barcelona)
susivarpe@hotmail.com

edad media de $32,26 \pm 7,92$ años, y el $81,5 \%$ son mujeres. El 88,9\% utilizó el modelo de Henderson en la escuela universitaria. Respecto al metaparadigma enfermero: en el concepto de Enfermería el 88,9\% se identificó con $\mathrm{V}$ Henderson; en el concepto de salud el $81,5 \%$ escogió la definición de Erickson; en el concepto de entorno el $63 \%$ se decantó por Henderson; y en el concepto de persona: el $70,4 \%$ señaló a Roy.

Se evidencia una gran variabilidad en cuanto al posicionamiento teórico de los profesionales. Además es necesario una mayor formación y reflexión del tema en nuestro ámbito.

PALABRAS CLAVE:

- MODELO DE ENFERMERÍA

- TEORÍA DE ENFERMERÍA

- CUIDADOS DE ENFERMERÍA

- ENFERMERÍA NEFROLÓGICA

\section{Nursing model and theory prospects in nephrology}

\section{Abstract}

Nursing depends on existence of a theoretic knowledge for everything areas. To use a conceptual model or nursing theory in the attention, guarantees that everybody nurses share equal metaparadigm conception and the same language in the cares guidance. Objectives are: 1. To investigate 
the nurses position of nephrology unit on conceptual models and nursing theories. 2. To investigate the application level on the same nursing models and theories.

It is a descriptive and observational study on all nurses of our unit. Data were capture by a questionnaire elaborate by us. Achieving a descriptive study of all variables, quantitative variables results were expressed as average and plus minus standard deviation. Categorical variables were expressed as frequency and percentages.

Twenty seven questionnaires were analysed. Response rate was $77.1 \%$. The demographic data are: average age $32.27 \pm 7.92$ years, and $81.5 \%$ are women. $88.9 \%$ used Henderson's model in the university school. Results nursing's metaparadigm: In the Nursing concept, $88,9 \%$ selected to V. Henderson; in the Health concept, $81.5 \%$ chose to Erickson; in the environment concept, $63 \%$ opted for Henderson; and in the person concept, $70.4 \%$ selected to Roy.

A wide variability of the about theoretic position of nurses was found. Also a robust training and analysis of the issue is necessary.

\section{KEY WORDS:}

NURSING MODEL

- NURSING THEORY

- NURSING CARE

NEPHROLOGY NURSE

\section{Introducción}

Enfermería como disciplina depende de la existencia de un conocimiento teórico para todas las áreas ${ }^{1,2}$. EI uso de un modelo Conceptual o Teoría de Enfermería en la atención garantiza que todas las enfermeras compartan igual concepción del metaparadigma e igual lenguaje en la orientación de los cuidados ${ }^{3}$. En este sentido, se trabaja a nivel nacional el proyecto NIPE (Normalización de las Intervenciones para la práctica Enfermera) que pretende establecer un lenguaje común. Utiliza el proceso de enfermería como núcleo de la metodología y recoge el paradigma enfermero ${ }^{4}$.
De mayor a menor nivel de abstracción los modelos y teorías de enfermería se dividen en metaparadigmas, filosofías, modelos conceptuales, grandes teorías, teorías y teorías de nivel medio. El metaparadigma es el nivel de conocimientos más abstracto de todos. Describe los principales conceptos que hacen referencia al tema principal, así como a la finalidad de una disciplina. Los principales conceptos del metaparadigma enfermero son el de persona, entorno, salud y enfermería.

Para que la aplicación en la práctica de un modelo de enfermería tenga éxito, es necesario que el profesional conozca el modelo teórico (conjunto de modelos abstractos), reflexione sobre éste (modelo mental) y finalmente lo aplique (modelo sustituto) ${ }^{5}$.

Según el desarrollo histórico de la enfermería, Kérouac $^{6}$ distingue 3 paradigmas:

Categorización (1850-1950): Los fenómenos son divisibles. Las relaciones que se establecen son lineales y causales. La enfermera suple las incapacidades, es la que tiene el conocimiento.

Integración (1950-1975): La persona es el eje central de la disciplina. La relación es circular e interaccional. La enfermera es consejera, ayuda a escoger los comportamientos de salud.

Transformación (1975- actualidad): El fenómeno se describe como único, complejo y global. La enfermera es partícipe junto a la persona de los cuidados.

Existe un gran número de enfermeras asistenciales que no encuentran aplicabilidad de los modelos teóricos en el trabajo diario ${ }^{7}$ y se preocupan por su habilidad técnica y el dominio de los procedimientos sin echar de menos la aplicación de una metodología para planificar los cuidados ${ }^{8}$. Probablemente por la alta tecnificación, los profesionales se encuentran en el paradigma de la categorización.

Existen varias justificaciones que defienden la pertinencia de la realización de este estudio:

a) En primer lugar, no se ha hallado bibliografía específica sobre el objeto de nuestro estudio (revisión en base de datos MEDLINE, Cuiden y en SEDEN). La mayoría de estudios realizados en este tema en Nefrología hablan de la aplicación 
de un modelo concreto al paciente nefrológico9,10, también se habla de modelos de gestión y organización de la sala de diálisis, y elaboración de protocolos. Se han encontrado estudios sobre la aplicabilidad del proceso de atención en los estudiantes de Enfermería.

b) Actualmente nuestro centro no dispone de un modelo de enfermería para la labor profesional, pero se halla en un momento de transición y desarrollo de una nueva metodología de trabajo de enfermería. Por esto, la actual investigación llega en un momento crucial del cambio, siendo pertinente el conocimiento previo de la filosofía de los profesionales de enfermería implicados.

c) Asimismo, nos ha permitido conocer la actitud de los profesionales de nuestra unidad respecto a las teorías de enfermería y comprobar si corrobora la afirmación de que "las enfermeras asistenciales no encuentran aplicabilidad de los modelos teóricos en el trabajo diario" hallada en la literatura revisada ${ }^{7}$ y ver si es diferente a la referenciada en la bibliografía específica de nefrología.

\section{Objetivos}

1. Conocer el posicionamiento de los profesionales de enfermería de la unidad de Nefrología sobre modelos conceptuales y teorías de enfermería.

2. Conocer el nivel de aplicabilidad sobre los modelos y teorías del cuidado.

\section{Material y Métodos}

Es un estudio descriptivo en el que se trata de retratar la realidad de la filosofía del cuidado en nuestro centro a través de un estudio observacional y forma parte de una investigación mayor realizada en todo el hospital (204 profesionales encuestados), en el cuál actualmente existe un cambio de metodología asistencial a través de la creación de un nuevo marco conceptual propio de los cuidados enfermeros.
Variables principales: modelos y teorías. Metaparadigma enfermero: concepto de enfermería, entorno, salud y persona.

Variables secundarias: sociodemográficas. Datos sobre formación y vida laboral. Tipo de registros. Búsqueda de información complementaria.

Recogida de datos: se utilizó un cuestionario de elaboración propia (revisado por el departamento de epidemiología del centro) que fue implementado durante el horario laboral del personal. Para probar el nivel de comprensión del mismo se realizó una prueba piloto pidiendo la colaboración a 10 profesionales de fuera de nuestra unidad. Se entregó en mano, previamente se realizó una sesión informativa. La recogida se realizó en una semana de plazo desde su implementación, mediante una urna para asegurar el anonimato.El cuestionario constaba de tres partes: carta de presentación, glosario de términos, y 37 preguntas (de las cuáles 36 eran cerradas de respuesta múltiple y una abierta). Las preguntas sobre el metaparadigma enfermero contenían enunciados sin identificar las autoras en que fueron basados estos enunciados.

Análisis estadístico: se realizó un estudio descriptivo de todas las variables descritas expresando los resultados de las variables cuantitativas en forma de media y desviación estándar. Las variables categóricas se expresaron en forma de frecuencia y porcentajes. Se utilizó el paquete estadístico SPSS 11.0.

Lugar de estudio: el presente trabajo se desarrolló en una unidad nefrológica hospitalaria dispone de 6 camas de agudos, 150 plazas de pacientes para HD y 30 plazas en diálisis peritoneal. Es el centro de referencia de once poblaciones con unos 400.000 habitantes. El personal de enfermería está formado por unas 48 personas de las cuáles 35 son enfermeras asistenciales.

Sujetos de estudio: la muestra estuvo formada por la totalidad de diplomados en enfermería y/o A.T.S, en activo en la unidad de Nefrología en el momento del estudio, y que quisieron participar de forma voluntaria.

Fueron solicitados los permisos de Dirección y del Comité de Ética de Investigación Científica. 


\section{Resultados}

Variables demográficas: se repartieron 35 cuestionarios, recogiéndose 27, con índice de respuesta del 77,1\%. Edad mínima 21 años, máxima 53, con una media de $32,26 \pm 7,92$ años. Un $81,5 \%$ son mujeres. La experiencia laboral de $10 \pm 8,31$ años, de los cuáles $8,48 \pm 6,51$ años en nuestro centro. El 92,6\% ha respondido el turno, siendo un $48,1 \%$ turno de mañana, un $29,6 \%$ turno tarde, un $7,4 \%$ a tiempo parcial, un 3,7\% turno de noche y un 3,7\% turno partido. Un $96,3 \%$ son DUE (diplomado universitario en enfermería), un 3,7\% son ATS (ayudante técnico sanitario) y no han convalidado su titulación.

Variables sobre la formación: el modelo que utilizaban en la escuela universitaria de enfermería se refleja en la figura 1 . Suma más del $100 \%$ porque hay profesionales que indicaron más de una teórica. Sólo el $11,1 \%$ del total ha realizado algún master / postgrado y/o algún curso relacionado con los modelos de enfermería. Además, cuando quieren ampliar conocimientos buscan información principalmente: en medicina, en fundamentos de enfermería y en farmacología.

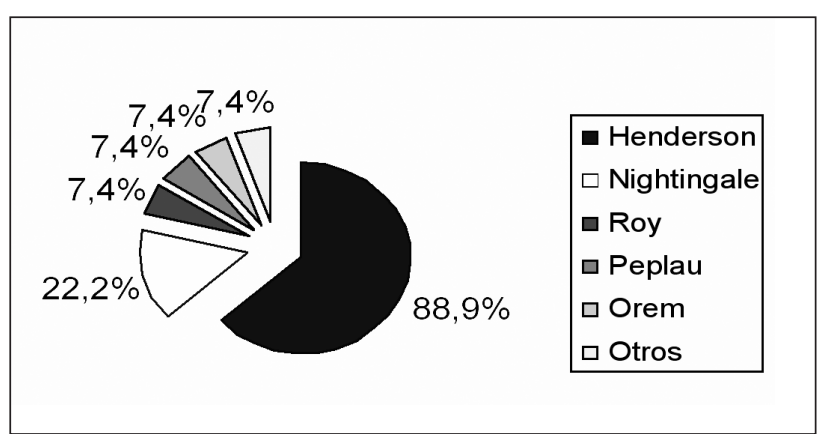

Figura 1: Modelo usado en la Escuela Universitaria de Enfermería.

Variables sobre el modelo: un 22,2\% afirma que sigue algún modelo en la actividad diaria (Henderson), un $66,7 \%$ contesta que no, y un $11,1 \%$ no lo sabe. Un $14,8 \%$ opina que siempre sigue el modelo que utilizaba como estudiante en las prácticas, un $29,6 \%$ no, un $55,6 \%$ a veces. Las respuestas respecto a la oportunidad de implantar un modelo se recogen en la figura 2; proponiendo el $44,4 \%$ a Henderson, el $3,7 \%$ a Nightingale, el $18,5 \%$ ningún modelo y el $33,3 \%$ no ha respondido. El 18,5\% opina que es necesario implantar diferentes modelos según las características del paciente, mientras que un $25,9 \%$ opina que no, un $44,4 \%$ a veces y un $11,1 \%$ no lo sabe. Al atender a la persona se identifican con un modelo conceptual: un $7,4 \%$ sí, siempre, un $18,5 \%$ casi siempre, un $63 \%$ a veces, un $11,1 \%$ nunca.

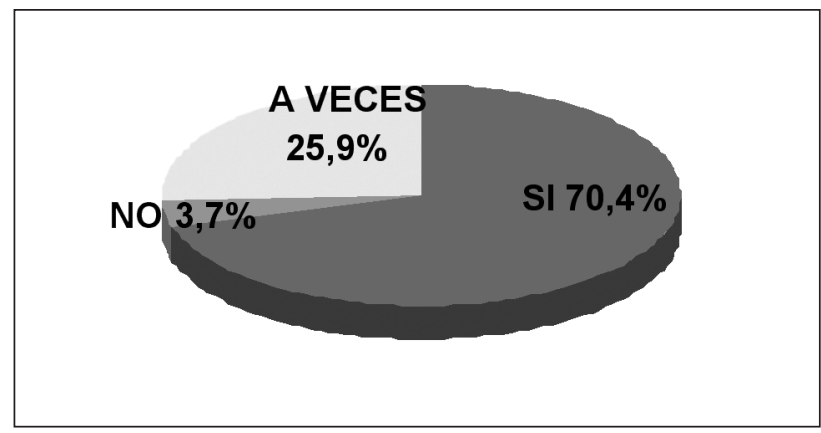

Figura 2: ¿Sería positivo implantar un modelo en nuestro centro?

Variables sobre los registros: el $63 \%$ cree que la hoja actual de registros no favorece el poder trabajar bajo un modelo de enfermería, mientras un $11,1 \%$ opina que sí, un $22,2 \%$ a veces. El $18,5 \%$ utiliza la hoja de valoración de enfermería al ingreso, mientras que un $51,9 \%$ no, un $29,6 \%$ a veces. El $37 \%$ opina que la hoja de valoración de enfermería al ingreso facilita el trabajo, mientras que un $25,9 \%$ no, un $29,6 \%$ a veces y un $7,4 \%$ no opina. Las observaciones que realizan y registran de forma habitual (pregunta con respuesta múltiple), un $88,9 \%$ el estado general, un $74,1 \%$ las curas, un $63 \%$ la alimentación, un $63 \%$ el grado de autonomía/dependencia, un $63 \%$ la higiene, un 59,3\% la movilización, un $55,6 \%$ la eliminación, un 51,9\% la relación con la familia, un $11,1 \%$ otras observaciones y nadie escoge hábitos religiosos.

Variables sobre el metaparadigma enfermero: en las cuestiones sobre el metaparadigma de la ciencia enfermera (enfermería, salud, entorno y persona), podían elegir diferentes enunciados basados en diversas autoras (en los cuestionarios no se mencionaba a las autoras) y hasta un máximo de 3 definiciones a la vez, de las cuáles escogieron:

- Concepto de enfermería: El 88,9\% se identificó con "Ayudar al individuo sano o enfermo en la realización de aquellas actividades que contribuyan a su salud o recuperación o a una muerte tranquila", basada en Henderson. El 66,7\% marcó "Profesión y disciplina humanista y científica que se centra en los cuidados para ayudar, apoyar, facilitar, o capacitar a las personas", derivada de Leininger. Además mencio- 
nar que el 22,2\% señaló la definición de Erickson, el $11,1 \%$ Rogers, el 7,4\% Orem, el Peplau, el 7,4\% King, el 3,7\% Nightingale, el 3,7\% Orlando y nadie se posicionó por Roy o por ninguna autora. El 81,5\% de los profesionales cree que enfermería es una profesión, un $11,1 \%$ un arte, un 7,4\% una vocación, nadie a marcado la opción de trabajo u otros.

- Concepto de salud: El 81,5\% escogió "Es un estado de bienestar físico, social y mental, y no únicamente la ausencia de enfermedad" basada en Erickson. El 37\% opinó que es "Ausencia de enfermedad, sensación de sentirse bien y la capacidad de usar al máximo todas las facultades de la persona", derivada de Nightingale. El $29 \%$ eligió la definición de Henderson, un 22,2\% Leininger, un $22,2 \%$ Trabelbee, un 3,7\% Benner, un 3,7\% Orem, un 3,7\% Roy, 3,7\% Levine y nadie escogió a Peplau, King, Rogers o ninguna autora.

- Concepto de entorno: El 63\% se decantó por "Conjunto de todas las condiciones e influencias externas que afectan a la vida y al desarrollo de un organismo", basada en Henderson. Un 55,6\% "Todas las condiciones y circunstancias que rodean y afectan el desarrollo y comportamiento de la persona", derivada de Roy. Un 25,9\% señaló el enunciado de Leninger, el 18,5\% Nightingale, el 11,1\% Levine, el 7,4\% Orem, el 3,7\% Peplau, nadie eligió a Rogers, Kolcaba o ninguna autora.

- Concepto de persona: El 70,4\% señaló "Es un ser biopsicosocial en constante interacción con el medio ambiente cambiante", basada en Roy. $Y$ un $40,7 \%$ "Ser humano que se encuentra en un proceso continuo de progreso, evolución y cambio", Travelbee. El 25,9\% escogió la derivada de Nightingale, el $11,1 \%$ Henderson, el $11,1 \%$ King, el 3,7\% Rogers, el 3,7\% Orlando 3,7\% Johnson, nadie marcó a Orem, Peplau, Wiedenbach o a ninguna teorética. En los registros de enfermería, al identificar a la persona, lo indican como el $74,1 \%$ paciente, el $18,5 \%$ el nombre, el $7,4 \%$ usuario, nadie indicó la opción de término cliente. La concepción del individuo para los profesionales es un 92,6\% holístico: biopsicosocial, el resto un ser unitario; nadie señaló ser que tiende a la máxima independencia, 0 un sistema estable que se puede desestabilizar, 0 un ser con requisitos de autocuidado.

\section{Discusión}

El personal es joven y con un nivel medio de experiencia, con poca formación Postgraduada en Modelos. La mayoría han sido formados bajo el modelo de Henderson, aunque no se identifican con este en los conceptos de salud y persona. Esto puede ser debido a que la práctica asistencial puede modificar la conceptualización enfermera. Además, por los resultados obtenidos se observa que no existe un paradigma dominante que clarifique las guías de pensamiento y práctica de la mayoría de los enfermeros/as, ya que la complejidad de las respuestas humanas centro del cuidado enfermero, dificulta que una sola definición pueda dar explicación a todas ellas ${ }^{11}$.

Un $30 \%$ considera que el modelo que usaba como estudiante no le es útil en la labor diaria, corroborando la bibliografía ${ }^{7,12}$. Cuando se atiende a la persona sólo un $7,4 \%$ lo hacen bajo en modelo con el cual se identifican. Esto puede estar relacionado con la ausencia de definición de un Modelo Enfermero en nuestro centro y quizás, por la tecnificación de la unidad de Nefrología 8 .

Sin embargo, un $70 \%$ de los profesionales sí desearían implantar un Modelo, aunque sólo un $48 \%$ se define por una autora en concreto, mayoritariamente coincide con la que se utilizó en su formación pregrado, quizás por el desconocimiento de otras Teorías. A menudo, los modelos y teorías de enfermería se relegan de la práctica enfermera porque son complejos y no se conocen en profundidad; sin embargo, es responsabilidad de todos los profesionales que la aplicación del cuidado tenga un buen fundamento teórico que permita el avance de la ciencia enfermera13. Se observan dudas si se deberían implantar diferentes modelos según las características de la persona, un $44,4 \%$ ha señalado a veces.

La mayoría de los profesionales considera que los documentos de registros actuales de Enfermería no favorecen trabajar bajo un modelo, posiblemente porque han quedado obsoletos. Aunque en centros donde tienen implantado un modelo (Henderson), reconocen que cumplimentan con poca frecuencia ${ }^{14}$.

Gran parte de los encuestados considera enfermería una profesión, dato que se diferencia con la encuesta de El Libro Blanco de Enfermería donde un gran nú- 
mero de profesionales se identificaba con vocación (Consejo General de Enfermería 1998) ${ }^{15}$.

Muy probablemente los profesionales eligen la definición de salud de Erickson porque esta autora utiliza la definición de la OMS, y es la más conocida mundialmente.

En las observaciones que realizan y registran de forma habitual nadie ha marcado la opción de hábitos religiosos, a pesar que el $92,6 \%$ dice que la persona es un ser holístico.

Todos los conceptos analizados del metaparadigma enfermero pertenecen mayoritariamente a autoras en el paradigma de la integración, esto se puede relacionar con que gran parte de los profesionales se han identificado con el término de paciente, la visión de la persona puede influir sobre el resto de los conceptos (salud, entorno, Enfermería). La visión actual de la persona dentro del ámbito hospitalario es todavía aquella que cree que la persona es un ser con necesidades, pero que aún no puede participar activamente en las acciones de su salud, se continua con una actitud algo paternalista. Se tiene interiorizado que es el eje central de nuestra atención pero aún se trabaja a partir de caminos paralelos, y no de forma conjunta, que sería lo deseable ante las nuevas demandas sociales (globalización, multiculturalidad). Hay que interiorizar el trabajar "con la persona" y no únicamente el "para la persona", o lo que es igual acabar de dejar el paradigma de la integración por uno más vigente, el de la transformación.

\section{Conclusiones}

1. Se evidencia una gran variabilidad en cuanto al posicionamiento teórico de los profesionales.

2. Debemos utilizar registros que reflejen la conceptualización Enfermera.

3. Es necesaria más formación sobre el tema.

4. Es un tema sobre el cuál se reflexiona poco en el ámbito de Nefrología.

\section{Bibliografía}

1. Marriner A, Raile M. Modelos y teorías en enfermería. 5a ed. Madrid: Mosby; 2003.

2. Potter y Perry. Fundamentos de enfermería. Fundamentos teóricos de la práctica enfermera. Volumen I. Madrid: Hartcourt - Mosby; 2002.

3. Campo MA, Oriach MR, Viladot A, Espinalt A, Fernández $C$. Servicio de cuidados enfermeros según modelo conceptual de Virginia Henderson. Implantación, resultados y costes. Metas Enferm 1999; 2(13):8-14.

4. ISICS. El proyecto NIPE: La normalización de la Práctica profesional. [acceso 26 0ct 2005]. Disponible en: http://www.nipe.enfermundi.com.

5. Wimpenny P. The meaning of models of nursing to practising nurses. J Adv Nurs. 2002; 40(3):346-54.

6. Kérouac S, Pepin J, Ducharme F, Duquette A, Major F. El pensamiento enfermero. Barcelona: Masson; 1996.

7. Medina JL. ¿Teoría? ¿práctica? El difícil equilibrio académico y profesional en la España "pre Europea" (II). Revista Rol Enfermería 2005; 28(4):246-248.

8. López J. Propuestas para el desarrollo profesional (I). Enferm Clin 2000; 10(3): 114-118.

9. Carmona M, García C, Salces R, Sanlés E, Pérez M. Estudio de caso bajo la aplicación de la teoría general del déficit de autocuidado (Modelo de autocuidados D. Orem) Rev Soc Esp Enferm Nefrol 2000; 10:36-38.

10. Fischer P. Plan de Cuidados de enfermería para enfermos renales siguiendo el modelo Krohwinkel. EDTNA-ERCA Journal 2002; 2:51-59.

11. Goulet $C$, et al. Enfermería de práctica avanzada: un tesoro oculto. Enferm Clin. 2003; 13(1):48-52.

12. Zaragoza A. El proceso de atención de Enfermería. Perspectiva de profesores y alumnos. Revista ROL Enfermería 1999; 22(9):583-590. 